\title{
Estudios de juventudes: una revisión de investigaciones en Argentina, Brasil y Colombia, 20II-20I9
}

\author{
Sara Victoria Alvarado, Ph.D. ${ }^{a}$ \\ Centro de Estudios Avanzados en Niñez y Juventud \\ Cinde-Universidad de Manizales, Colombia \\ Pablo Vommaro, Ph.D. ${ }^{b}$ \\ Universidad de Buenos Aires, Argentina
}

\author{
Jhoana A. Patiño, Mag. ${ }^{\complement}$ \\ Centro de Estudios Avanzados en Niñez y Juventud \\ Cinde-Universidad de Manizales, Colombia \\ Silvia H. S. Borelli, Ph.D. ${ }^{d}$ \\ Universidad Católica de São Paulo, Brasil
}

\section{Resumen (analítico)}

Se presenta una revisión documental sistemática de estudios de juventudes en Argentina, Brasil y Colombia entre el año 2011 y el 2019. Los resultados describen las condiciones de vida en las dimensiones social, cultural y política de las juventudes e identifican las tendencias investigativas en los estudios de juventudes en estos tres países. La discusión muestra que construcción social, política y cultural de las juventudes en la Argentina, Brasil y Colombia es entendida desde tres elementos: las condiciones de desigualdades y violencias estructurales en las que despliegan y producen sus vidas las y los jóvenes, una intensificación de las pluralidades y multiplicidades como marca generacional y la consolidación del sujeto juvenil como protagonista de la vida política y el conflicto social en contextos contemporáneos.

\section{Palabras clave}

Investigación, Argentina, Colombia, Brasil.

\section{Thesauro}

Tesauro de Ciencias Sociales de la Unesco.

\section{Para citar este artículo}

Alvarado, S. V., Vommaro, P., Patiño, J. A., \& Borelli, S. H. S. (2021). Estudios de juventudes: una revisión de investigaciones en Argentina, Brasil y Colombia, 2011-2019. Revista Latinoamericana de Ciencias Sociales, Niñez y Juventud, 19(2), 1-25.

https://dx.doi.org/10.11600/rlcsnj.19.2.4545

\section{Historial}

Recibido: 14.09.2020

Aceptado: 18.11.2020

Publicado: 27.04.2021

\section{Información artículo}

El presente artículo de revisión documental se realiza en el marco de cuatro proyectos de investigación realizados por los autores, en la Argentina, Brasil y Colombia entre el 2012 y el 2020 (los cuales se detallan dentro del artículo). Área: Socioloaía. 
Youth Studies: A Review of Research in Argentina, Brazil, and Colombia, 20II-20I9

Abstract (analytical)

This paper is based on a systematic documentary review of youth studies carried out in Argentina, Brazil and Colombia between 2011 and 2019. The results of these studies describe the living conditions experienced by young people in the social, cultural and political dimensions of their lives, and also identify research trends in youth studies in these three countries. The discussion shows that the social, political and cultural construction of youth in Argentina, Brazil and Colombia can be under-stood through three elements: conditions of inequality and structural violence in which young people live, an intensification of pluralities and multiplicities as a generational marker, and consolidation of the youth subject as the protagonist of political life and social conflict in contemporary contexts.

Keywords: Research, Argentina, Colombia, Brazil.

Authors' Keywords: Youth, social construction, cultural construction, political construction.

Estudos sobre juventudes: uma revisão de pesquisa na Argentina, Brasil e Colômbia, 2OII-2OI9

Resumo (analítico)

Trata-se de um artigo, baseado em revisão documental e sistemática de estudos sobre juventudes em Argentina, Brasil e Colômbia, entre os anos 2011 e 2019. Os resultados analisam as condições de vida das juventudes nos aspectos social, cultural e político, e identificam as tendências investigativas dos estudos sobre juventudes nesses três países. Resultam dessa reflexão, três elementos para a compreensão da construção social, política e cultural das juventudes na Argentina, Brasil e Colômbia: as condições de desigualdades e violências estruturais nas quais os jovens se desenvolvem e produzem suas vidas, a intensificação das pluralidades e multiplicidades como marco geracional e a consolidação do sujeito juvenil como protagonista da vida política e do conflito social nos contextos contemporâneos.

Palavras-chave: Pesquisa, Argentina, Colômbia, Brasil.

Palavras-chave autores: Juventudes, construção social, construção cultural, construção política.

Información autores

[a] Psicóloga, Universidad Javeriana. Magíster en Educación y Desarrollo Social. Doctora en Educación, Nova University-Cinde; Posdoctora en Ciencias Sociales, Niñez y Juventud de la Universidad de Manizales, Cinde, Universidad Católica de Sao Paulo, Clacso. Directora del Centro de Estudios Avanzados en Niñez y Juventud Cinde-Universidad de Manizales. (iD) 0000-0002-0115-8075. H5: 18. Correo electrónico: s.v.alvarado.s@gmail.com

[b] Historiador. Posdoctor en Ciencias Sociales, Niñez y Juventud (PUC-SP, Colef, U. Manizales/Cinde, UNLa, Clacso). Doctor en Ciencias Sociales y Profesor de Historia de la Universidad de Buenos Aires. Profesor de Historia (UBA). Investigador Adjunto del Conicet y del Instituto de Investigaciones Gino Germani (UBA). H5: 20. Correo electrónico: pvommaro@gmail.com (iD) 0000-0002-6957-0453.

[c] Magíster en Educación y Desarrollo Humano. Candidata a Doctora en Ciencias Sociales, Niñez y Juventud, Cinde-Universidad Manizales. Investigadora asociada ante Minciencias. (ID) 0000-0002-8431-7317. H5: 8.

Correo electrónico: alexaebano@gmail.com

[d] Antropóloga. Doctora, Libre Docente y Profesora de la Facultad de Ciencias Sociales y del Programa de Estudios de Posgrado en Ciencias Sociales, de la Pontificia Universidad Católica de São Paulo (PUCSP. Brasil). H5: 21. (iD) 0000-0003-3510-6625. Correo electrónico: siborelli@gmail.com 


\section{Introducción}

1 presente artículo resulta de un ejercicio cualitativo de revisión documental en
el marco de cuatro investigaciones sobre juventudes —en Argentina, Brasil y Colombia - y vinculadas al grupo de trabajo Clacso Juventud e infancia: prácticas políticas y culturales, memorias y desigualdades en el escenario contemporáneo.

Desde estas investigaciones, los autores abordaron —con perspectivas diversas- a las juventudes en contextos situados. Desde ellas se configuraron corpus documentales de importancia analítica que fueron retomados como base para desarrollar una lectura sistemática de las condiciones de vida de las juventudes en estos tres países y de las tendencias en las investigaciones sobre juventudes. En este sentido, a continuación se muestra de manera resumida el marco de las cuatro investigaciones con el fin de situar de forma general a los lectores.

La primera investigación es La cultura como vector de protección: participación de niños, niñas, adolescentes y jóvenes en 5 regiones del país, codirigida por Silvia H. S. Borelli (Arregui et al., 2020) desde la Coordinación de Estudios y Desarrollo de Proyectos Especiales (PUCSP). Este proyecto se propuso identificar y analizar las formas en que se procesa la vida cultural de la niñez, la adolescencia y la juventud en los espacios de decisión de las políticas públicas, así como la actuación de colectivos más autónomos, marcando su condición de sujetos de derechos y resaltando la importancia de la cultura como instrumento privilegiado de mediación para las prácticas políticas, protección, interacción y participación social. El objetivo fue desarrollar una perspectiva dialógica y participativa con estos sujetos, sus producciones y formas de apropiación en cinco regiones de Brasil y en cinco estados de la Federación: São Paulo (sudeste), Bahía (nordeste), Pará (norte), Rio Grande do Sul (sur) y Distrito Federal (medio oeste). El proyecto apostó por una metodología cualitativa y participativa que buscó incluir a los niños, las niñas, adolescentes y jóvenes en la construcción del conocimiento y en la identificación y debate de formas culturales que promuevan la acción colectiva. La propuesta buscaba así dialogar 
con el carácter inseparable de la promoción, protección y defensa de los derechos y la ampliación del conocimiento y la difusión de información. Se realizó una revisión documental, relevamiento y mapeo de acciones socioculturales y colectivas, una investigación etnográfica en línea (cuestionario, entrevistas en profundidad y grupos de discusión en foros virtuales con adolescentes/jóvenes, colectivos y organizaciones) y círculos de conversación presenciales con niños y niñas.

La segunda investigación desde la que planteamos este artículo es la desarrollada en la Argentina por Pablo Vommaro, desde el Grupo de Estudios de Políticas y Juventudes (Universidad de Buenos Aires), denominada Activismo y compromiso político juvenil: un estudio socio-histórico de sus experiencias políticas y militantes (1969-2011), realizada entre 2014-2018 y financiada por la ANPCyT. En esta se estudiaron las transformaciones del activismo juvenil en este periodo, tanto desde una mirada diacrónica (a través de la cual se abordaron las formas de adhesión y participación juvenil a lo largo del tiempo) como desde una perspectiva sincrónica, por medio de la cual se dio cuenta de la diversidad de grupos, posiciones, formas de participar y de dar sentido a la política en un mismo momento del tiempo. Se apostó por una estrategia metodológica cualitativa que postulaba la importancia de comprender el sentido de la acción humana para la explicación de los procesos sociales. Se procuró recuperar la perspectiva de los participantes, que habían interpretado sus propias acciones y les habían atribuido un significado, para inscribir estas narrativas en un marco de inteligibilidad mayor constituido por fuentes secundarias que emergieron del propio trabajo de campo (materiales de las propias organizaciones y relevamiento de prensa, provistos por las mismas personas entrevistadas), así como también la bibliografía acumulada en los períodos. El enfoque escogido para la investigación tomó como objeto del análisis los itinerarios o carreras militantes antes que los grupos o colectivos de los cuales formaban parte los y las jóvenes activistas, para desarrollar una lectura que pusiera en relación el contexto sociohistórico, el tiempo colectivo y el personal, dimensiones ineludibles en el análisis del activismo desde un punto de vista biográfico. Se hicieron entrevistas semiestructuradas y revisión documental de fuentes periodísticas, incluyendo los documentos producidos por organizaciones, colectivos, grupos o acciones de protesta (prensas, folletos, declaraciones, cuadernillos de formación, páginas web, blogs, etc.) en las que se encontraban involucrados los jóvenes activistas.

En tercera instancia trabajamos desde la investigación Jóvenes militantes y espacios juveniles en agrupaciones político partidarias: una aproximación a las formas de compromiso juvenil luego de la crisis de 2001, realizada entre 2015-2018 y codirigida por Pablo Vommaro, también 
desde el Grupo de Estudios de Políticas y Juventudes de la Universidad de Buenos Aires. La investigación buscó analizar la manera en que se construyó y resignificó la categoría joven en espacios políticos partidarios luego de la crisis del año 2001 y hasta la actualidad. Se estudiaron las transformaciones acontecidas durante estos años, indagando las formas de militancia política y las concepciones acerca de la política generadas a partir del impulso de espacios juveniles asociados a las formas político partidarias, en general, y de aquellos vinculados con la gestión pública estatal en particular. Metodológicamente la investigación asumió una aproximación sociohistórica y sociopolítica que permitió analizar la construcción de la juventud como un objeto político, dando cuenta de su proceso de constitución y evitando la reificación del mismo. En lugar de considerar a la juventud como noción que remite a un grupo de edad o como momento en un ciclo de vida de las personas, el proyecto analizó la génesis de la juventud como categoría política, los diferentes usos que se hacen de la misma, las disputas por el uso legítimo e ilegítimo de este término, así como el potencial movilizador de adhesiones y las prácticas militantes asociadas a esta. El análisis de la construcción de lo juvenil dentro de los partidos políticos se realizó atendiendo al entramado de relaciones sociales cotidianas vinculadas con su formación y sostenimiento. Se trabajó con casos, se hicieron talleres y grupos focales, entrevistas a profundidad y entrevistas biográficas.

Finalmente, la cuarta investigación es Infancias y juventudes latinoamericanas: violencias, conflictos, memorias y procesos de construcción de paz realizada en coautoría por Sara Victoria Alvarado desde el grupo Perspectivas políticas, éticas y morales de la niñez y la juventud de la Universidad Manizales y el Cinde. Tuvo como propósito aportar a la comprensión de la constitución de los niños, niñas y jóvenes en contextos de violencias. Tanto desde elementos macro — que involucran la historia y las dinámicas de nación-como desde procesos relacionales en los cuales la violencia se naturaliza como parte de la vida social. Interesó comprender, no solo la afectación frente a la violencia, sino principalmente los modos en los que los niños, niñas y jóvenes emergen como sujetos políticos capaces de aportar a la construcción de paz, con el fin de identificar experiencias y caminos posibles en los procesos de socialización política y formación ciudadana de niños, niñas y jóvenes, y la generación de nuevas maneras de distribución del poder más cercanas a la democracia. El estudio fue de carácter mixto, incorporando a nivel cuantitativo el análisis estadístico de una encuesta en línea que fue contestada por los niños, niñas y jóvenes en la institución educativa; $y$, a nivel cualitativo, una metodología hermenéutica ontológica política orientada a las comprensiones de los sentidos y las prácticas alrededor de los procesos de socialización, de los que participan niños, niñas y jóvenes en contextos 
de vulnerabilidad y violencia, y a la transformación que propenden por el agenciamiento de los niños, niñas y jóvenes como sujetos políticos. Para ello se realizaron talleres lúdico-creativos y grupos focales en los cuales se ampliaron algunos sentidos y prácticas emergentes en la investigación.

En el marco de estas investigaciones, realizamos la construcción de este artículo de revisión desde dos preguntas centrales: la primera es ¿cuáles son las condiciones de vida que marcan las juventudes contemporáneas de Argentina Brasil y Colombia?, y, la segunda, ¿cuáles son las tendencias de las investigaciones que se han realizado en Argentina, Brasil y Colombia en el campo de estudios de las juventudes durante los últimos 20 años?

\section{Método}

Para dar cuenta de estas preguntas optamos por un método cualitativo de revisión documental de textos. Entre marzo y diciembre de 2019 realizamos una revisión general de los más de zoo textos que ya habían sido seleccionados por los equipos de investigación de los cuatro proyectos que dan base a este artículo, y que previamente habían sido identificados en bases de datos como ProQuest, Scopus, SciElO, Clacso, Periódicos Capes, SIB/Dédalus y Google Académico, por medio de las palabras claves: construcción social, juventudes, acción colectiva juvenil, condición de las juventudes, prácticas políticas juveniles, contexto de las juventudes y resistencia de las juventudes (tanto en español como en portugués).

Tras la lectura inicial, se seleccionaron 8o textos para conformar el corpus analítico de este artículo; este contiene investigaciones empíricas, investigaciones relacionadas con los temas abordados elaboradas por otros equipos e investigadores e informes de agencias nacionales e internacionales respecto a las condiciones de vida de las juventudes. Además de lo anterior, la configuración de este corpus cumplió con los siguientes criterios: a) investigaciones e informes publicados especialmente entre el año 2011 y el 2019; b) investigaciones relacionadas con los procesos de construcción social, política y cultural de las juventudes latinoamericanas y, especialmente, en los tres contextos de análisis; c) interesadas en analizar las desigualdades estructurales en las que viven las juventudes; d) que tuvieran algún componente relacionado con el análisis de la participación social y política de las juventudes en movilizaciones recientes. 
Luego de la selección del corpus para este análisis cualitativo documental, acudimos a las fichas analíticas que ya habían desarrollado los equipos investigativos de los cuatro proyectos, con el fin de extraer de ellas la información relacionada con las preguntas de revisión. Para el caso de las fichas en las que no se encontraba información detallada sobre nuestras preguntas de estudio documental, volvimos sobre el texto directamente para hacer la lectura y extraer lo requerido. Posteriormente, organizamos la información por países; elaboramos unas matrices descriptivas que permitieron identificar cronologías, tendencias y similitudes. Finalmente, pasamos a la etapa de escritura de resultados comparando las tres matrices y ordenando las comprensiones a partir de las preguntas iniciales.

\section{Resultados}

\section{Condiciones de vida que marcan la construcción de las} juventudes contemporáneas de Argentina, Brasil y Colombia

En la Argentina es necesario destacar cuatro escenarios de producción social de las juventudes argentinas en tiempos de crisis. En primer lugar, al comienzo del período vemos un incremento de la degradación de las condiciones de vida de las juventudes, con aumento de índices de pobreza, desempleo y abandono escolar, entre otros, lo que configura una situación de desigualdades entramadas y crecientes. Así, el desempleo juvenil subió, del $13 \%$ a comienzos de los años noventa, a más del 36\% a comienzos del presente siglo (Centro de Economía Política Argentina [Cepa], 2017); en una triplicación que acompañó a otros indicadores que empeoraron como la tasa de escolarización o la de pobreza.

En segundo lugar, una relativa mejora de indicadores entre 2003 y 2015, con un nuevo deterioro entre 2015 y 2019. Por ejemplo, la tasa de pobreza juvenil pasó, de menos del $30 \%$ en 2010, a más del $37 \%$ en 2017 (Observatorio de la Deuda Social Argentina de la UCA, 2017). En cuanto al desempleo juvenil, bajó del 36.9\% en 2003, a menos del 20\% en 2013 y volvió a subir al $22 \%$ en 2016 (Cepa, 2017).

En tercer término, en el período abierto luego de 2001, no solamente se reactualizan los usos y apropiaciones de la categoría juventud(es), sino que además se constata tanto la emergencia como la revitalización de espacios juveniles en el marco de movimientos, colectivos y partidos políticos preexistentes (Vázquez \& Vommaro, 2012; Vázquez et al., 2018). Así, los estudios llevados a cabo desde el Grupo de Estudios de Políticas y Juventudes del Instituto de Investigaciones Gino Germani de la Universidad de Buenos Aires 
permiten identificar que en 2008 se configura otro ciclo de movilización e ingreso a la militancia juvenil (Vázquez et al., 2018).

En estos años podemos observar que la militancia juvenil aumenta su visibilidad pública a través de grupos que destacan la juventud de sus miembros y se autoidentifican a partir de la dimensión generacional, así como también se constata la exaltación de la juventud como atributo positivo por parte del mundo adulto (Vázquez \& Vommaro, 2012). Esto se produce en una coyuntura en la cual el Estado recompone su capacidad de gestión y de centralizar la política formal e institucionalizada (Vázquez et al., 2018). ${ }^{1}$ Es decir, que entre los años 2008 y 2015 cobró forma un período en el observamos la reemergencia y relegitimación de formas clásicas de participación (por ejemplo, espacios político-partidarios o sindicales), en las que las juventudes habían tenido menor protagonismo en años anteriores.

Así mismo, entre 2008 y 2015 se experimentó un proceso de ampliación de derechos y reconocimiento de las diversidades juveniles (Vommaro, 2015). El género, las sexualidades, las diversidades y disidencias, así como los derechos y reconocimientos que a estas se vinculan fueron una causa militante de creciente importancia en el período. A la lucha por el matrimonio igualitario (logrado en 2010), se sumó la movilización por la identidad de género aprobada en 2012. Unos años más tarde emergió el movimiento Ni una menos, que ocupó calles y plazas visibilizando una lucha de años, ahora protagonizada por nuevas generaciones juveniles, sobre todo femeninas. Estos movimientos crecieron y se multiplicaron con expresiones como «Vivas nos queremos», el paro de mujeres y, en los últimos años, las luchas por la interrupción legal del embarazo y el aborto seguro, legal y gratuito. Este protagonismo juvenil y femenino fue tematizado como «revolución de las hijas en» medios de comunicación y ámbitos académicos por autoras como Elizalde (2018, 2019).

En Brasil, el autoritarismo, la disolución de un modelo de sociedad centrado en la integración de contingentes subordinados y la defensa de los derechos humanos tuvieron su origen en 2016, con la destitución de la presidenta Dilma Roussef. El cargo lo ocupó el vicepresidente Michel Temer (2016-2018), luego de negociaciones políticas entre las fuerzas más conservadoras del país. El autoritarismo alcanzó su punto máximo con la investidura del presidente Jair Bolsonaro en 2019.

\footnotetext{
${ }^{1}$ Nos referimos a la presidencia de Néstor Kirchner (2003-2007) y a las dos presidencias de Cristina Fernández de Kirchner (2007-2011 y 2011-2015).
} 
Los jóvenes se vieron afectados por estas condiciones e inestabilidades políticas, sociales y económicas, por las incertidumbres que han erradicado los derechos y logros sociales adquiridos y por la disolución acelerada de las políticas públicas dirigidas a la juventud. Políticas afirmativas que fueron parte de la historia entre 2003-2015, implementadas por gobiernos que priorizaron la articulación entre políticas sociales y desarrollo económico, y se consolidaron a través de acciones inclusivas e intersectoriales, que tuvieron como objetivo la integración y defensa de los derechos juveniles.

En 2018, la población brasileña se estimó en 208494900 habitantes y, de este total, 47.3 millones eran jóvenes entre 15 y 29 años (Instituto Brasileiro de Geografía e Estatística, 2018, p. 12). Si bien los indicadores educativos en Brasil arrojaron resultados positivos entre 2004-2012, es necesario alertar sobre a precariedad de las condiciones educativas y laborales reveladas: el 7.6\% de los jóvenes, entre 15 y 24 años, son analfabetos; el 23\% no asiste a la escuela y está excluido del mercado laboral (p. 2); y bajo el título «color o raza» (personas de 15 años o más), la diferencia es notablemente desigual: mientras que el $9.1 \%$ de analfabetos está entre las personas de color negro o marrón, solo el $3.9 \%$ lo está entre las personas de color blanco. Sin embargo, lo que más destaca en el conjunto de indicadores sobre la juventud en Brasil es la violencia resultante de múltiples feminicidios, juvenicidios (Valenzuela, 2012, 2015) y etnocidios (Castro, 2017).

El Atlas de la violencia (Instituto de Pesquisa Econômica Aplicada [Ipea], 2019, p. 25) informa que, del total de jóvenes asesinados en 2017, el 94.4\% eran hombres. «El 59.1\% del total de muertes de hombres entre 15 y 19 años son causadas por homicidios» (p. 6) y «los homicidios son la principal causa de muerte de los jóvenes: $51.85 \%$ de 15 a 19 años; $49.4 \%$ entre 20 y 24; $38.6 \%$ entre 25 y 29» (p. 25). Aunque los hombres son los más afectados, y la mayor concentración de defunciones se da a los 21 años (p. 7o), las mujeres representan un total del $41.7 \%$, distribuidas entre los 18 y los 30 años. Además de los juvenicidios, los resultados apuntan a un aumento de la «violencia letal» contra públicos específicos: en 2017, del total de homicidios, el 75.5\% fueron personas negras (Ipea, 2019, p. 49).

La violencia letal que afecta a las personas LGBTQI+ es más difícil de contabilizar, debido a la «invisibilidad desde el punto de vista de la producción oficial de datos» (Ipea, 2019, p. 56). Sin embargo, el informe Colombia diversa (2019) destaca a Brasil como uno de los más responsables de la muerte violenta de personas LGBTQI+, e informa que más de 1300 personas lesbianas, gais, bisexuales, transexuales e intersexuales han perdido la vida de forma violenta en los últimos cinco años. También revela que «la mayoría de los 
delitos que involucran a hombres gay, bisexuales y transgénero, así como a lesbianas y mujeres transexuales, ocurrieron entre los 18 y 25 años y entre los 26 y 30 años» (p. 5).

En Colombia, la precariedad atraviesa la vida de las juventudes. Según el Departamento Nacional de Estadística (Dane, 2019), de los 48 millones de habitantes que tiene el territorio, 12 millones (es decir, aproximadamente el 27\%) son jóvenes entre 14 y 28 años, y tres millones habitan en zonas rurales con condiciones de vida altamente precarias y desiguales, especialmente, para los jóvenes indígenas, campesinos, gitanos y habitantes en zonas de frontera, con mayor incidencia en las mujeres.

En la Cumbre de Juventud (realizada por el Banco Mundial en 2016) se mostró que en Colombia existían aproximadamente 6.3 millones de jóvenes activos en el mercado laboral. La tasa de desempleo para los hombres fue $13.7 \%$, mientras que para las mujeres se ubicó en $22.4 \%$. En cuanto al acceso a los servicios públicos de los jóvenes, el Dane (2019) identificó que en el censo nacional del 2018, en las viviendas donde ellos habitan, un $4 \%$ no tienen acceso a energía eléctrica, el $14 \%$ no tiene acueducto, el $24 \%$ no cuenta con agua, el $34 \%$ no está conectada a redes de gas y el $47 \%$ no cuenta con acceso a internet. Según datos de la ONU (2017), la condición de vivienda de las mujeres jóvenes en sectores rurales es 3 veces inferior a la de los hombres jóvenes. Y dentro de las mujeres rurales, las indígenas y afros afrontan mayores niveles de pobreza y exclusión en sus viviendas. A nivel de educación, las tasas de deserción son altas (Sistema para la Prevención de la Deserción de la Educación Superior, 2019).

Respecto al impacto de la violencia social, el Instituto Nacional de Medicina Legal (2019) informó que, a noviembre de 2019, habían ocurrido 10468 homicidios, un $2.34 \%$ más que en igual período del año pasado. La mayor cantidad de homicidios se ubicó en la franja de 20 a 24 años, con 2072 casos, seguida por la de 25 a 29 años con 1878 casos, configurándose una situación de juvenicidio. En cuanto a las violencias de género, el Observatorio de Feminicidios de Colombia (2019) expuso que, hasta septiembre de 2019, se registraron más de 400 casos, en los cuales el $40 \%$ de las víctimas eran mujeres entre los 14 y 28 años de edad.

Sobre el impacto de los hechos victimizantes del conflicto armado en los trayectos biográficos de los jóvenes colombianos, el Registro Único de Víctimas de la Unidad para la Atención y Reparación Integral a las Víctimas (2019), informó que hubo 1800 ooo jóvenes desplazados, de los cuales, más de un millón eran mujeres; 44 oooo mil jóvenes fueron víctimas de desaparición forzada, 24 ooo mil fueron hombres y 20000 mil mujeres; 21000 mil fueron víctimas en atentados, hostigamientos y acciones terroristas; 5400 fueron 
víctimas de minas antipersonas y 13 ooo jóvenes padecieron violencia sexual (más del $90 \%$ de los casos se presentó en mujeres).

\section{Tendencias de los estudios de las juventudes en Argentina, Brasil y Colombia y su lectura en torno a la construcción social, política y cultural de las juventudes}

Estudios de juventudes en Argentina en los últimos diez años

Ante la gran profusión de estudios de juventudes que se produjo en la Argentina luego de 2008 , en este apartado nos centraremos en tres líneas y espacios que expresan y condensan este proceso de expansión del campo.

En primer lugar, la conformación de la Red Nacional de Investigadoras/es en Juventudes de la Argentina (ReIJA) en 2004, que celebró su primera reunión en 2007 y ya realizó seis encuentros, el último en Córdoba en 2018. Este es un espacio que articula a casi trescientos investigadores sobre juventudes de la Argentina y que tiene vinculaciones internacionales. Recorriendo las actas de las reuniones de esta Red (denominadas Renija) es posible identificar énfasis y emergencias temáticas y renovaciones generacionales. Por ejemplo, entre 2007 y 2018 el número de grupos se amplía, pasando de 10 a 17, con la emergencia de temas como: experiencias de trabajo con jóvenes, desigualdades, jóvenes y memoria, cuerpos, ruralidades y migraciones.

En segundo lugar, destacamos los trabajos que se enfocan en la participación y el activismo juvenil en ámbitos relacionados con los partidos políticos, el Estado y las formas institucionales. Aquí mencionamos los estudios sobre juventudes vinculadas con experiencias partidarias dentro del kirchnerismo, así como en fuerzas políticas de un amplio espectro político-ideológico (Campos \& Segura, 2012; Mutuverría, 2011; RoccaRivarola, 2016; Vázquez, 2010, 2015; Vázquez \& Vommaro, 2012; Vommaro et al., 2015).

En otros trabajos se exploran las particularidades que reviste la incorporación de militantes al Estado (Perelmiter, 2016); en especial, las relaciones entre la militancia juvenil partidaria y gestión en la administración pública estatal (Vázquez, 2015; Vázquez \& Cozachcow, 2017; Vázquez et al., 2018). Así mismo, se revitaliza el interés por la comprensión de los procesos de invención de las juventudes en términos sociopolíticos, nacionales e identitarios (Kriger, 2016).

Dentro de estas temáticas se han desarrollado trabajos que abordaron la participación estudiantil en centros de estudiantes secundarios (Enrique, 2011; Larrondo, 2014) y 
universitarios (Blanco, 2016; Bonavena \& Millán, 2012; Carli, 2012; Kandel, 2005); los procesos vinculados con la sanción del denominado «voto joven» (Blanco et al., 2014; Vommaro, 2015); la revitalización del activismo juvenil al interior del espectro sindical (Galimberti, 2016; Wolanski, 2016); la politización del género, la sexualidad y la intimidad (Blanco, 2014a, 2014b; Elizalde, 2018) y la movilización colectiva en contra del denominado «gatillo fácil», la persecución, el hostigamiento y el accionar represivo del Estado hacia las juventudes (Bonvillani, 2015; Schaefer, 2018). También podemos mencionar trabajos que analizan la formulación de discursos sociales y mediáticos que tienden a fijar relaciones entre las condiciones de joven pobre y joven delincuente (Gentile, 2015) y los conflictos con la ley penal y criminalizaciones que experimentan (Daroqui \& Guemureman, 2014; Galano, 2019; Guemureman, 2015).

Los abordajes desde la institucionalidad y las políticas públicas de juventudes se manifiestan en investigaciones que abordan casos provinciales o municipales (Balardini, 2008), así como también programas nacionales (Chaves et al., 2016; Miranda \& Miguel, 2018; Vázquez, 2015).

En tercer término, destacamos la colección «Las juventudes argentinas hoy: tendencias, perspectivas, debates», que reúne 34 libros publicados entre 2015 y 2020 acerca de las problemáticas y experiencias de los y las jóvenes en la Argentina actual en los distintos espacios en los que despliegan sus vidas: escuelas, barrios, redes sociales, lugares de trabajo y de esparcimiento, ámbitos culturales y políticos, sin olvidar a aquellos que se encuentran en conflicto con los organismos de seguridad estatales y están institucionalizados o sometidos a un estado de permanente sospecha, vigilancia y hostigamiento (Vommaro, 2015).

\section{Tendencias de los estudios de juventudes en Brasil}

El balance de la producción sobre jóvenes en Brasil, entre 2010 y 2019 (Borelli et al., 2020), ${ }^{2}$ se puede dividir en tres ejes principales. El primero, centrado en documentos e informaciones relacionados con la fundamentación y sistematización de políticas sociales, producidos en la esfera pública, como, por ejemplo, la Secretaria Nacional da Juventude (2010), el Conselho Nacional de Juventude (2010) y el Fondo de Población de las Naciones Unidas (2010). Además, es posible observar la producción de algunas reflexiones enfocadas en el diálogo crítico en torno a la consolidación de estas políticas, con énfasis

\footnotetext{
${ }^{2}$ Acceso a la información completa resultante de la encuesta «Producción académica sobre juventudes en Brasil (2010-2019)»: https://bit.ly/3nlWEI3
} 
en la desigualdad social, los derechos humanos, la educación, los movimientos sociales, la participación, las vulnerabilidades y la violencia. Entre otros, cabe mencionar los trabajos de M. Abramovay, S. H. S. Borelli, M. G. Castro, J. T. Dayrell, R. Novaes, R. C. A. Oliveira y M. Sposito. ${ }^{3}$ Es posible notar, en un contexto en el que las políticas generaron resultados y se conquistaron derechos, un giro en el debate centrado en las propias políticas hacia el predominio de una mirada centralizada en los usos que los jóvenes hacen de las prerrogativas logradas.

Las políticas públicas se analizan, por ejemplo, desde la perspectiva de la demanda de ampliación de la participación política, a través de redes de sociabilidad y proyectos de vida; por la emancipación; por la democracia; por el logro de más ciudadanía; para la extensión de los derechos humanos; la expansión de la inclusión (a través de la educación y las prácticas educativas/pedagógicas, presencial o en redes digitales, trabajo, cultura, ocio, comunicación); y las violencias. De manera relevante, autores como H. Abramo, M. G. Castro, J. T. Dayrell, Instituto Pólis, R. Novaes, J. J. Waiselfisz, entre otros. ${ }^{4}$

El segundo eje presenta publicaciones centradas en la relación entre jóvenes, política, medios de comunicación, cultura y urbanidades vinculadas a acciones colectivas y prácticas políticas juveniles en las ciudades, la producción y consumo cultural y mediático, las prácticas de resistencia mediadas por la música y otros intervenciones artístico-culturales y la fuerte presencia del movimiento hip hop. En ellos, las acciones culturales se configuran en un lugar privilegiado de las acciones políticas y las dimensiones estéticoculturales como fuertes indicadores para la comprensión de las prácticas políticas juveniles en la época contemporánea: M. Abramovay, C. C. Arregui, S. H. S. Borelli, M. G. Castro, J. T. Dayrell, M. Herschmann, Instituto Pólis, R. Novaes, R. C. A. Oliveira, S. L. Pereira, R. L. M. Rocha y M. Sposito. ${ }^{5}$

Y, finalmente, un tercer eje centrado en producciones sobre violencia y exclusiones alusivas a estigmas y prejuicios raciales y de género en su interseccionalidad: condiciones sociales, socialización y comunicación relacionadas con el género; cuerpo y sexualidad; legalización del aborto; movimiento negro y promoción de la igualdad racial; cuotas e identidades culturales en la diáspora; vidas de migrantes: M. Abramovay, M. G. Castro, J. T. Dayrell, Colectivo de Militancia Investigadora, C. C. Garcia, N. M. M. Gusmão, Insti-

\footnotetext{
3 https://bit.ly/3sSVtzX

${ }^{4}$ Ídem.

5 Ídem.
} 
tuto Pólis, R. L. Soares, R. C. A. Oliveira, R. Recuero, R. L. M. Rocha, entre otros. ${ }^{6}$ Es de destacar que las publicaciones relacionadas con este eje se vieron impactadas, en particular, por un hecho brutal: el asesinato de la concejala de Río de Janeiro, Marielle Franco, en marzo de 2018. Esto revela la dimensión interseccional de la exclusión de clase, raza y género. Ser mujer, negra, lesbiana y con un liderazgo periférico significa ocupar un papel protagonista en el contexto de una crónica de muerte anunciada.

Tendencias de los estudios de política-juventud y de las prácticas políticas de las juventudes en Colombia

Según Cubides et al. (1998, retomando a Perea, 1997), los estudios de juventud en Colombia han tenido tres períodos. Un primer momento denominado la juventud imaginaria entre los años 1940 y 1950, en el cual el país vivió la polarización partidista y los jóvenes fueron incluidos dentro de la política como objeto pasivo de guerra. La relación política-juventud estaba mediada por la concepción de patria y por su expresión formal. En un segundo momento, nombrado como la juventud subversiva y comprendido entre 1950 y 1980 , cuyo contexto sociopolítico estuvo marcado por el reacomodamiento demográfico de alta migración del campo a la ciudad y la masificación de la educación como facilitadora de la emergencia de un tipo de joven que confrontaba el orden existente y se cohesiona en organizaciones estudiantiles de carácter nacional. Por último, un tercer momento llamado la juventud sin máscara, el cual se ubica desde la década de los ochenta hasta la actualidad, marcado por la intensificación de la violencia y el auge del narcotráfico y sicariato, con un papel protagónico de la juventud urbana marginada.

Según Alvarado et al. (2010), en Colombia, la relación entre política y juventud se ha abordado investigativamente desde una perspectiva histórica en dos líneas: trabajos historiográficos y cronológicos y estudios comprensivos y reconstructivos.

Por otra parte, Ospina et al. (2011), Agudelo-Ramírez et al. (2013) y Patiño et al. (2014) consideran que los estudios de juventud a nivel de Latinoamérica y Colombia pueden analizarse en dos tendencias. La primera de ellas se refiere a una comprensión estadocéntrica y formal de la vinculación de los y las jóvenes a los sistemas tradicionales de participación política, tales como el voto y la participación en partidos políticos. La segunda, alude al carácter sociocéntrico y cultural de las subjetividades e identificaciones y movilizaciones de los jóvenes.

6 Ídem. 
De manera relacional y ampliada, García et al. (2018) sostienen que los estudios sobre jóvenes y acción política en Colombia pueden agruparse en tres tipos: un primer tipo caracterizado por el análisis de la organización de los jóvenes, desde una visión de política centrada en el asunto de la lucha por el poder y de la participación partidista y de clase, dentro de lo cual se asumen diferentes visiones sobre los jóvenes como problema, como víctima o como encarnación de valores. Un segundo tipo lo constituyen estudios que proponen otras miradas sobre lo joven, desde su relación con la cultura, la identidad, la sensibilidad juvenil y los procesos de cambio que caracterizan a los jóvenes. Y, en un tercer tipo, los estudios se interesan, en forma directa, por la subjetividad política de los jóvenes, sus modos de agrupamiento y sus formas de participación, cuyas expresiones son predominantemente estéticas, mediadas por las tecnologías de la información y comunicación.

\section{Discusión}

A partir de los resultados descritos desde la revisión documental, entendemos que la construcción social, cultural y política de las juventudes se da en una coyuntura contemporánea de globalización, en la que se deja ver la creciente producción y reproducción de las desigualdades multidimensionales. Estas desigualdades están ancladas, en particular, en las condiciones de subordinación experimentadas por poblaciones marginalizadas y subalternizadas como niños, adolescentes, jóvenes y ancianos; por mujeres sometidas al régimen patriarcal y sexista; por afros, negros e indígenas estigmatizados y excluidos por diferentes racismos; por personas LGBTQI+ segregadas y amenazadas; por migrantes y refugiados, originarios de los múltiples desplazamientos que cruzan los continentes; por los habitantes de las periferias de las grandes ciudades, que siguen en conflicto disputando urbanidades y por los habitantes y los ocupantes que viven en la confrontación entre la metrópolis modulada, desinfectada y mercantilizada y los territorios y comunidades habitados desde las resistencias y re-existencias de la vida cotidiana.

Por otra parte, los estudios y experiencias analizadas muestran que en situaciones de desigualdades y violencias como las que se despliegan en países como Argentina, Colombia y Brasil emergen las movilizaciones sociales, los levantamientos populares, las mingas indígenas, las resistencias territoriales y comunitarias, las huelgas de trabajadores y las calles desbordadas de mujeres, estudiantes y personas LGBTQI+, donde convergen las voces de quienes luchan por el reconocimiento, por no ser negados en su condición de diferencia y alteridad, por su derecho a existir y re-existir. Esto se expresa en un prota- 
gonismo cada vez más relevante de las juventudes, que signan las dinámicas de los conflictos sociales y las acciones colectivas en la región.

A partir de esto, aparece la necesidad de pensar el presente de estas juventudes desde las maneras como son producidas social y estatalmente. Es decir, desde la maquinaria del Estado que produce a los jóvenes como grupo social precarizado, estigmatizado y segregado, como objeto del consumo, como mercancía para la guerra, como enemigo, amenaza o peligro, como descarte. Pero también desde las múltiples expresiones de las resistencias, que afirman a los jóvenes como sujetos políticos, colectivos y organizados, como estética de la existencia, como imaginación sublevada, como lo común en la diferencia o como paisaje insurrecto (Reguillo, 2017).

Las investigaciones aquí revisadas en clave de estudios acerca de las juventudes latinoamericanas muestran que no basta leer la condición juvenil anclada a las teorías del futuro ni solo a las condiciones del presente posible. Pensar la condición juvenil y las configuraciones generacionales en el paisaje de las confrontaciones de América Latina requiere orientar la mirada hacia otros focos de experiencia en los cuales se disputan los sentidos de las juventudes pluralizadas y diversas.

De acuerdo a lo anterior, postulamos que la construcción social, política y cultural de las juventudes en la Argentina, Brasil y Colombia, a la luz de estos estudios descritos en este artículo, puede ser entendida desde cuatro elementos: en primer lugar, las condiciones de desigualdades multidimensionales en las que despliegan y producen sus vidas las y los jóvenes de los tres países seleccionados. Estas desigualdades, de las que aquí destacamos la dimensión generacional, constituyen uno de los principales elementos a tener en cuenta a la hora de explicar y comprender las múltiples violencias - materiales y simbólicas-que experimentan las juventudes en la actualidad en interseccionalidad con dimensiones como las sexo-genéricas, las territoriales y las socioeconómicas. Las realidades analizadas en este trabajo coinciden con las estadísticas producidas por la ONU (2017), donde se señala que existen en el mundo 1.800 millones de jóvenes entre los $10 \mathrm{y}$ 24 años de edad, encontrándose el $40 \%$ de ellos en precarias condiciones de vida. Para América Latina y el Caribe, según datos de 2012, las personas de entre 15 y 29 años son 156 millones, de los cuales casi un $30 \%$ pueden ser considerados pobres, porcentaje que aumentó en los últimos años y se magnificó con la pandemia de COVID-19.

En segundo lugar, una intensificación de las pluralidades y multiplicidades, entendidas como marca generacional y no en clave de fragmentación o dispersión. Es decir, juventudes construidas desde la diferencia poniendo de relieve las diversidades como una de 
las características distintivas a la hora de desentrañar los procesos de su producción social. Diferencia entendida desde la potencia y la capacidad de producir lo común en la diversidad, de instituir otras formas de estar juntos desde la pluralidad y la polifonía. Estos modos diversos de ser joven en Colombia, Argentina y Brasil, cruzados por las condiciones de desigualdades multidimensionales y las formas de producción estatal subalternizadoras y adultocéntricas, producen procesos de estigmatización, desacreditación o aniquilamiento identitario que signan y condicionan los espacios de vida de las y los jóvenes, sobre todo los de barrios populares y territorios marginalizados.

En tercer término, se identifica un proceso de consolidación del sujeto juvenil como protagonista de la vida política y el conflicto social en los tres países seleccionados y en la región en general. No exentos de cambios, discontinuidades y tensiones, los modos de participación, militancia y movilización que produjeron los jóvenes en los últimos años signaron la dinámica del proceso político a nivel territorial, nacional y regional, instituyendo propuestas y modos de ser que desafían lo instituido e interpelan a otros sectores y grupos sociales. Así, identificamos formas persistentes, resistentes y emergentes de compromiso público juvenil, desafiando los modos legitimados y consagrados, y mostrando alternativas y aperturas que provocan disrupciones e insinúan otros caminos posibles.

Por último, en cuarto lugar, en la revisión bibliográfica realizada pudimos identificar que, al calor del protagonismo juvenil creciente en la vida social de cada uno de los países analizados y en la región en general, en las últimas décadas se consolidó un campo de estudios de juventudes que expresó estas transformaciones y buscó interpretarlas. Así, asistimos a una ampliación de los estudios de juventudes y a una expansión de los espacios académicos para desarrollarlos. En el mismo sentido, en algunos países como Argentina y Brasil (hasta 2015) esta expansión y consolidación del campo de estudios de juventudes coincidió con un momento de crecimiento de las políticas públicas de juventudes (al menos de las sectoriales), concebidas incipientemente de modo transversal e integral. Se generaron así interesantes iniciativas de articulación y retroalimentación entre academia y políticas públicas que se vieron interrumpidas por los cambios de gobierno sucedidos en 2015-2016, a partir de sucesos de diversa índole en ambos países. 


\section{Conclusiones}

Dentro de la actual coyuntura de globalización, Brasil, Colombia y Argentina están incluidos en el sello global países emergentes - al menos desde un punto de vista económico-, a la vez que las relaciones sociopolíticas establecidas desde el Estado hacia la sociedad civil - y particularmente hacia las juventudes-, se caracterizan por diferentes rasgos de autoritarismo y violencias, con singularidades y coyunturas específicas en cada caso.

Lo que más se destaca para Brasil, Argentina y Colombia en el conjunto de características y dimensiones presentadas sobre las juventudes desde los estudios revisados en este artículo son las violencias resultantes de múltiples estigmatizaciones, racismos, feminicidios, juvenicidios, etnocidios y el asesinato de líderes sociales; estos se han convertido en las víctimas preferidas de un sistema clasista, racista, patriarcal, sexista y homofóbico. Así mismo, la precarización de las vidas juveniles se ha convertido en la característica que marca su vida cotidiana en las grandes ciudades y en las zonas rurales, conf1gurando mundos subalternizados y degradados a partir de los cuales las juventudes producen sus existencias y sus resistencias generacionalmente configuradas.

Concluimos también que, en los tres países seleccionados y en otros de América Latina, las juventudes se movilizan por causas no necesariamente juveniles o juvenilistas. Así, los movimientos que protagonizan las y los jóvenes interpelan y emergen de problemáticas más amplias que incluyen a otros sectores sociales, complejizando el carácter (inter)generacional de estos procesos y mostrando que el mundo de los jóvenes es plural y se encuentra atravesado por diversidades, interseccionalidades e intersectorialidades de género, clase social, etnia y territorio, entre otras.

Lo que se puede llamar «participación juvenil» en el siglo XXI tiene que ver, básicamente, con la acción social constitutiva de la condición juvenil contemporánea. Pero no cualquier tipo de acción. Se trata de una acción colectiva juvenil de resistencia a lo establecido, de fuga, de invención, de sustracción y éxodo (Vommaro, 2015). Es la agencia entendida como el poder de actuar diferente (Muñoz, 2012, p. 31). Una acción colectiva que genera sospechas, enrarece el mundo, inventa tramas y que no busca mantener los privilegios históricamente constituidos sino promover la creación, la resistencia, la transformación social, ampliar derechos, lograr reconocimientos e instituir formas otras de vida y existencia.

Así entonces, en condiciones de precarización y subalternizaciones, pero inventando y recreando resistencias, ocupando calles y barrios y desplegando alternativas, mane- 
ras de decir basta y proponer otros caminos, construyen sus vidas las juventudes latinoamericanas. Como señala Lazzarato (2006), la radicalidad novedosa de los comportamientos políticos de las juventudes de hoy puede verse en la práctica de una lógica que vindica la diferencia y la asume como motor de la cooperación; en la articulación en un nosotros común que no se fusiona en un todo unívoco, homogéneo y armónico; en la acción política sustentada en los acontecimientos y no en proyectos políticos unívocos y lineales y en la experiencia cotidiana de acciones efímeras, diversas, contingentes y disruptivas, más que desde la configuración de sujetos y grupos con identidades fijas o estables.

\section{Referencias}

Agudelo-Ramírez, A., Murillo, L., Echeverry-Restrepo, L., \& Patiño-López, J. A. (2013). Participación ciudadana y prácticas políticas de jóvenes en la cotidianidad. Revista Latinoamericana de Ciencias Sociales, Niñez y Juventud, 11(2), 587-602.

Alvarado, S. V., Botero, P., \& Ospina, H. F. (2010). Subjetividades políticas: sus emergencias, tramas y opacidades en el marco de la acción política. Mapeo de 61 experiencias con vinculación de jóvenes en Colombia. Utopía y Praxis Latinoamericana, 15 (50), 39-55.

Arregui, C. C., Borelli, S. H. S., \& Pontual, P. C. (2020). Cultura como vetor de proteção: protagonismo de crianças e adolescentes. Educ. https://bit.ly/2QU1K4X

Balardini, S. (2008). De deejays, floggers y ciberchabones: subjetividades juveniles y tecnocultura. En R. Bendit, M. Hahn, \& A. Miranda (Comps.), Los jóvenes y el futuro. Prometeo.

Blanco, R. (2014a). Universidad íntima y sexualidades públicas: la gestión de la identidad en la experiencia estudiantil. Miño y Dávila.

Blanco, R. (2014b). Estudiantes, militantes, activistas: nuevas agendas de las agrupaciones universitarias en torno al género y la diversidad sexual. Perfiles Educativos, 36(144), 140-156. https://doi.org/10.22201/iisue.24486167e.2014.144.46018

Blanco, R. (2016). Escenas militantes: lenguajes, identidades politicas y nuevas agendas del activismo estudiantil universitario. Clacso.

Blanco, R., Larrondo, M., Núñez, P., Vázquez, M., \& Vommaro, P. (2014). Revisitando la década del 8o: una lectura sobre la participación juvenil en los albores de la recuperación democrática argentina. IV Reunión Nacional de Investigadores en Juventudes de la República Argentina. 
*Blanco, R., \& Vommaro, P. (2018). Otros caminos, otros destinos: transformaciones en los espacios y prácticas cotidianas de participación juvenil en los años ochenta. En M. Vázquez, P. Vommaro, P. Núñez, \& R. Blanco (Comps.), Militancias juveniles en la Argentina democrática: trayectorias, espacios y figuras de activismo (pp. 1-26). Imago Mundi.

Bonavena, P., \& Millán, M. (2012). El movimiento estudiantil en la actualidad argentina: una aproximación socio-histórica. Osal, XIII(31), 105-122.

Bonvillani, A. (2015). El Código de Faltas de la Provincia de Córdoba (Argentina) como dispositivo de poder: la construcción de la seguridad a partir de la equivalencia simbólica «joven pobre=peligroso». Revista Controversias y Concurrencias Latinoamericanas, $7(11), 81-101$.

*Borelli, S. H. S., \& Aboboreira, A. (2011). Teorias/metodologias: trajetos de investigação com coletivos juvenis em São Paulo/Brasil. Revista Latinoamericana de Ciencias Sociales, Niñez y Juventud, 1(9), 161-172.

Borelli, S. H. S., Klaus, P., Queiroz, A. (2020). Produção bibliográfica sobre juventudes, 2010-2019. https://www.pucsp.br/projetojovensurbanos/downloads/publicacoes/2Estado_da_Arte_Prod_Biblio_Juvent_2010-2019_Nov2o.pdf

*Borelli, S. H. S., Oliveira, R. A., Rangel, L. H. V., \& Rocha, R. M. (2010). Jovens urbanos, ações estético-culturais e novas práticas políticas: estado da arte (1960-2000). En S. V. Alvarado, \& P. A. Vommaro (Orgs.), Jóvenes, cultura y política en América Latina: algunos trayectos de sus relaciones, experiencias y lecturas (1960-200o) (pp. 293-324). Homo Sapiens; Clacso.

Campos, H., \& Segura, D. (2012). El rol de los y las jóvenes en las organizaciones kirchneristas de Santiago del Estero. Red de Investigadores/as en Juventudes de Argentina.

Carli, S. (2012). El estudiante universitario: hacia una historia del presente de la educación pública. Siglo XXI.

Castro, E. V. (2017). Sobre a noção de etnocídio, com especial atenção ao caso brasileiro. https://bit.ly/3euNapW

*Cazachcow, A. (2013). Jóvenes y partidos políticos en la Argentina actual: reflexiones acerca de los sentidos construidos por jóvenes en torno a su participación en partidos políticos. Sociedad Argentina de Análisis Político; Universidad Nacional de Entre Ríos.

Centro de Economía Política de Argentina. (2017, 1 de junio). Jóvenes y empleo en números. https://centrocepa.com.ar/informes/162-jovenes-y-empleo-en-numeros.html

Colombia Diversa. (2019). El prejuicio no conoce fronteras: homicidios de lesbianas, gay, bisexuales, trans e intersex en países de América Latina y el Caribe, 2014-2019. https:// sinviolencia.lgbt/wp-content/uploads/2019/o8/Informe_Prejuicios_compressed.pdf 
Conselho Nacional de Juventude. (2010). Conselhos de juventude: fortalecendo diálogos, promovendo direitos. https://bibliotecadigital.mdh.gov.br/jspui/handle/192/72

Cubides, H. J., Laverde, M. C., \& Valderrama, C. E. (1998). Viviendo a toda: jóvenes, territorios culturales y nuevas sensibilidades. Fundación Universidad Central; Siglo del Hombre.

*Chaves, M. (2009). Investigaciones sobre juventudes en la Argentina: estado del arte en ciencias sociales 1983-2006. Papeles de Trabajo, 2(5), 37-62.

Chaves, M., Fuentes, S., \& Vecino, L. (2016). Experiencias juveniles de desigualdad: fronteras y merecimientos en sectores populares, medios altos y altos. Grupo Editor Universitario.

*Chaves, M., \& Núñez, P. (2012). Juventud y política en la Argentina democrática: la invención de tradiciones, la creación de nuevas tendencias (1983-2008). Young. Nordic Journal of Youth Research, 20(4), 357-376.

Daroqui, A., \& Guemureman, S. (2014). Registro de violencias padecidas por los jóvenes en el sistema carcelario: las micro penalidades y los suplementos punitivos. Revista Diálogos Possíveis, (28), 206-236.

Departamento Nacional de Estadística. (2019). Boletín técnico mercado laboral de la juventud. Autor.

Elizalde, S. (2018). Hijas, hermanas, nietas: genealogías políticas en el activismo de género de las jóvenes. Revista Ensambles en Sociedad, Política y Cultura, (4), 86-93.

Elizalde, S., \& Romero, G. (2019). Cuerpos, emocionalidad y sentidos disruptivos en rituales juveniles de celebración escolar. Textura. Revista de Educação e Letras, 21(47), 132-154. https://doi.org/10.17648/textura-2358-0801-21-47-5090

Enrique, I. (2011). La participación estudiantil en la escuela secundaria en la Argentina: reconstrucción del conflicto en torno al protagonismo político de los jóvenes [Tesis de Maestría]. Universidad de Buenos Aires.

Fundo de População das Nações Unidas. (2010). Direitos da população jovem: um marco para o desenvolvimento. http://www.unfpa.org.br/Arquivos/direitos_pop_jovem.pdf Galano, N. (2019). Muerte joven y vidas precarias: notas sobre el juvenicidio. En D. Beretta, F. Laredo, P. Nuñez, \& P. Vommaro (Eds.), Políticas de juventudes y participación política (pp. 229-248). Universidad Nacional de Rosario.

Galimberti, C. (2016). Sindicatos y representaciones sobre el desarrollo regional en la Juventud Sindical Peronista Regional La Plata, Berisso y Ensenada (2010-2015) [Tesis de Maestría]. Universidad Nacional de La Plata.

García., C. N., Velásquez, N., Marín., V., Ocampo, O., \& Bassi, G. (2018). Estado del arte sobre el conocimiento producido en torno a la movilización y acción política de jóvenes en 
América Latina (Colombia, Chile, Uruguay, Argentina, Bolivia y México). Cinde; Universidad Manizales.

Gentile, F. (2015). Genealogía de las figuras de la infancia y juventud problemática en la Argentina. Universidad de Buenos Aires.

Guemureman, S. (2015). Adentro y afuera: juventudes, sistema penal y políticas de seguridad. Grupo Editor Universitario.

Instituto Brasileiro de Geografia e Estatística. (2018). Pesquisa Nacional por Amostra de Domicílios Contínua. Educação. https://bit.ly/3nrpTcy

Instituto de Pesquisa Econômica Aplicada. (2019). Atlas da violência. https://bit.ly/zaIL6cD

Instituto Nacional de Medicina Legal. (2019). Forensis. https://bit.ly/32PMojb

Kandel, V. (2005). Participación estudiantil y gobierno universitario: nuevos actores-viejas estructuras [Tesis de Maestría] Facultad Latinoamericana de Ciencias Sociales.

Kriger, M. (2016). La tercera invención de la juventud: dinámicas de la politización juvenil en tiempos de la reconstrucción del Estado-Nación (Argentina, 2002-2015). Grupo Editorial Universitario; Clacso.

Larrondo, M. (2014). Después de la noche. Participación en la escuela y movimiento estudiantil secundario: Provincia de Buenos Aires, 1983-2013 [Tesis de Doctorado]. Universidad Nacional de General Sarmiento-Ides.

*Larrondo, M., \& Cozachcow, A. (2018). Un llamado a la unidad: la experiencia del Movimiento de Juventudes Políticas (Mojupo) en la transición a la democracia. En M. Vázquez, P. Vommaro, P. Núñez, \& R. Blanco (Comps.), Militancias juveniles en la Argentina democrática: trayectorias, espacios y figuras de activismo. Imago Mundi.

Lazzarato, M. (2006). Políticas del acontecimiento. Tinta Limón.

*Miranda, A. (2015). Sobre la escasa pertinencia de la categoría NI NI: una contribución al debate plural sobre la situación de la juventud en la Argentina contemporánea. Revista Latinoamericana de Políticas y Administración de la Educación, 2(3), 60-73.

Miranda, A. \& Miguel, P. (2018). Políticas y leyes de primer empleo en América Latina: tensiones entre inserción y construcción de trayectorias. Revista de Ciencias Sociales, 31(42), 79-106. https://doi.org/10.26489/rvs.v31i42.4

Muñoz, G. (2012). ¿Qué significa ser joven en Colombia hoy? En F. Rojas, G. Muñoz, \& L. Corredor (Eds.), Jóvenes \& adultos: una pedagogía del encuentro (pp. 54-69). Editorial Pontificia Universidad Javeriana.

Mutuverría, M. (2011). Jóvenes en partidos políticos de La Plata: una mirada sobre los estudios de los jóvenes y la práctica política. Questión, 1(30), 1-7. 
Nateras, A. (2015). El aniquilamiento identitario infanto-juvenil en Centroamérica: el caso de la Mara Salvatrucha (MS-13) y la «pandilla» del barrio 18 (B-18). En J. M. Valenzuela (Coord.), Juvenicidio: Ayotzinapa y las vidas precarias en América Latina y España (pp. 99-13o). NED; El Colegio de la Frontera Norte.

Observatorio de Feminicidios de Colombia. (2019). Vivas nos queremos: septiembre 2019. https://bit.ly/3vmqPBu

Observatorio de la Deuda Social Argentina de la UCA. (2017). Pobreza y desigualdad por ingresos en la Argentina urbana 2010-2017. https://bit.ly/3aYC4IT

Organización de las Naciones Unidas. (2017). Informe anual 2017-2018. https://bit.ly/32R839a Ospina, H. F., Alvarado, S. V., Botero, P., Patiño, J., \& Carona, M. (2011). Experiencias alternativas de acción política con participación de jóvenes en Colombia. Cinde; Universidad de Manizales.

Patiño, J., Alvarado, S. V., Ospina-Alvarado, M. C. (2014). Ampliación de sentidos sobre las prácticas políticas de jóvenes con vinculación a siete movimientos sociales en Colombia. Revista Latinoamericana de Ciencias Sociales, Niñez y Juventud, 12(1), 257-275.

Perelmiter, L. (2016). Burocracia plebeya: la trastienda de la asistencia social en el Estado argentino. Universidad Nacional de San Martín.

Reguillo, R. (2017). Paisajes insurrectos: jóvenes, redes y revueltas en el otoño civilizatorio. NED Ediciones.

Rocca-Rivarola, D. (2016). La Cámpora movilizada: observación participante y reflexiones sobre la militancia oficialista durante el segundo gobierno de Cristina Fernández de Kirchner (2011-2015). Sures, (7), 1-22.

*Rocca-Rivarola, D. (2019). Militar en el gobierno: generaciones de militancia juvenil en los gobiernos argentinos y brasileros (2003-2015/2016). Grupo Editor Universitario.

Schaefer, V. (2018). La participación política en jóvenes organizados contra la violencia policial: el caso del colectivo de jóvenes por nuestros derechos de la ciudad de Córdoba (Argentina). Última Década, 26(49), 166-188. https://doi.org/f8qv

Secretaria Nacional da Juventude. (2010). Guia de políticas públicas de juventude. https:// bibliotecadigital.mdh.gov.br/jspui/handle/192/156

Sistema para la Prevención de la Deserción de la Educación Superior. (2019). Informe anual. Ministerio de Educación de Colombia.

Unidad para la Atención y Reparación Integral a las Víctimas. (2019). Registro Único de Victimas. https://bit.ly/3ase 815

Valenzuela, J. M. (2012). Sed de mal: feminicidio, jóvenes y exclusión social. El Colegio de la Frontera Norte; UANL. 
Valenzuela, J. M. (2015). Juvenicidio: Ayotzinapa y las vidas precarias en América Latina. NED; El Colegio de la Frontera Norte.

Vázquez, M. (2010). Experiencia social y militancia política: los referentes jóvenes de un movimiento de trabajadores desocupados. En A. Massetti, E. Villanueva, \& E. Gómez (Comps.), Movilizaciones, protestas e identidades politicas en la Argentina del bicentenario. Nueva Trilce.

Vázquez, M. (2015). Juventudes, políticas públicas y participación: un estudio de las producciones socioestatales de juventud en la Argentina reciente. Grupo Editor Universitario. https:// doi.org/10.2307/j.ctvtxw2nk

Vázquez, M., \& Cozachcow, A. (2017). Activismo juvenil en partidos con gestiones de gobierno a nivel subnacional en Argentina (2007-2015). Revista de Sociología e Política, 25(64), 47-72. https://doi.org/10.1590/1678-987317256405

Vázquez, M., \& Liguori, M. (2018). La gestión estatal de juventud durante la vuelta a la democracia en Argentina: agendas, escenarios y actores (1982-1987). Encrucijadas Revista Crítica de Ciencias Sociales, (15), 1-23.

*Vázquez, M., \& Núñez, P. (2013). «Organizarnos para transformar»: entre el impulso de políticas públicas integrales de juventud y la construcción de una juventud militante. Alas.

Vázquez, M., Rocca-Rivarola, D., \& Cozachcow, A. (2018). Fotografías de las juventudes militantes en Argentina: un análisis de los compromisos políticos juveniles en el Movimiento Evita, el Partido Socialista y el PRO entre 2013 y 2015. En M. Vázquez, P. Vommaro, P. Núñez, \& R. Blanco (Coords.), Militancias juveniles en la Argentina democrática: trayectorias, espacios y figuras de activismo. Imago Mundi.

Vázquez, M., \& Vommaro, P. (2012). Con la fuerza de la juventud: aproximaciones a la militancia kirchnerista desde La Cámpora. En G. Pérez, \& A. Natalucci (Comps.), Vamos las bandas: organizaciones y militancia kirchnerista. Nueva Trilce.

Vázquez, M., \& Vommaro, P. (2015). Entre la inclusión y la participación: una revisión de las políticas públicas de juventud en la Argentina actual. En H. Cubides, R. Unda, S. Borelli, \& M. Vázquez (Eds.), Juventudes latinoamericanas: prácticas socioculturales, políticas y políticas públicas. Cinde; Universidad de Manizales; Clacso.

Vázquez, M., Vommaro, P., Núñez, P., \& Blanco, R. (Comps.) (2018). Militancias juveniles en la Argentina democrática: trayectorias, espacios y figuras de activismo. Imago Mundi.

*Vommaro, P. (2010). Política, territorio y comunidad: las organizaciones sociales urbanas en la zona sur del Gran Buenos Aires (1970-200o) [Tesis doctoral]. Facultad de Ciencias Sociales; Universidad de Buenos Aires. 
*Vommaro, P. (2013). Balance crítico y perspectivas acerca de los estudios sobre juventudes y participación política en la Argentina (1960-2012). Sudamérica: Revista de Ciencias Sociales, (2), 91-130.

Vommaro, P. (2015). Juventudes y políticas en la Argentina y en América Latina: tendencias, conflictos y desafíos. Grupo Editor Universitario.

*Vommaro, P. (2017). Juventudes latinoamericanas: diversidades y desigualdades. Revista Temas, (87-88), 4-11.

Vommaro, G., Morresi, S., \& Bellotti, A. (2015). Mundo Pro: radiografía de un partido fabricado para ganar. Planeta.

Wolanski, S. (2016). Las nuevas generaciones del sindicalismo: jóvenes, trabajo y organización gremial en la Argentina. Grupo Editor Universitario. 